\title{
Integrating microRNA expression, miRNA-mRNA regulation network and signal pathway: a novel strategy for lung cancer biomarker discovery
}

\author{
Renqing Nie ${ }^{1}$, Wenling Niu ${ }^{1}$, Tang Tang ${ }^{1}$, Jin Zhang ${ }^{1}$, Xiaoyi Zhang ${ }^{\text {Corresp. } 1}$ \\ ${ }^{1}$ Faculty of Environment and Life, Beijing University of Technology, Beijing, China \\ Corresponding Author: Xiaoyi Zhang \\ Email address: zhangxiaoyi@bjut.edu.cn
}

Background. Since there are inextricably connections among molecules in the biological networks, it would be a more efficient and accurate research strategy to screen microRNA (miRNA) markers combining with miRNA-mRNA regulatory networks. The independent regulation mode is more "fragile" and "influential" than the co-regulation mode. miRNAs can be used as biomarkers if they can independently regulate hub genes with important roles in the PPI network, simultaneously the expression products of the regulated hub genes play important roles in the signaling pathways of related tissue diseases.

Methods. We collected miRNA expression of non-small cell lung cancer (NSCLC) from The Cancer Genome Atlas (TCGA) database and the Gene Expression Omnibus (GEO) database. Volcano plot and signal-to-noise ratio (SNR) methods were used to obtain significant differentially expressed (SDE) miRNAs from the TCGA database and GEO database, respectively. A human miRNA-mRNA regulatory network was constructed and the number of genes uniquely targeted (NOG) by a certain miRNA was calculated. The Area Under the Curve (AUC) values were used to screen for clinical sensitivity and specificity. The candidate markers were obtained using the criteria of the top five maximum AUC values and NOG $\geq 3$. The protein-protein interaction (PPI) network was constructed and independently regulated hub genes were obtained. Gene Ontology (GO) analysis and KEGG pathway analysis were used to identify genes involved in cancer-related pathways. Finally, the miRNA which can independently regulate a hub gene and the hub gene can participate in an important cancer-related pathway was considered as a biomarker. The AUC values and gene expression profile analysis from two external GEO datasets as well as literature validation were used to verify the screening capability and reliability of this marker.

Results. 15 SDE miRNAs in lung cancer were obtained from the intersection of volcano plot and SNR based on the GEO database and the TCGA database. Five miRNAs with the top five maximum AUC values and NOG $\geq 3$ were screened out. 61 hub genes were obtained from the PPI network. It was found that the hub gene GTF2F2 was independently regulated by miR-708-5p. Further pathway analysis indicated that GTF2F2 participates in protein expression by binding with polymerase II, and it can regulate transcription and accelerate tumor growth. Hence, miR-708-5p could be used as a biomarker. The good screening capability and reliability of miR-708-5p as a lung cancer marker were confirmed by AUC values and gene expression profiling of external datasets, and experimental literature. The potential mechanism of miR-708-5p was proposed.

Conclusions. This study proposes a new idea for lung cancer marker screening by integrating microRNA expression, regulation network and signal pathway. miR-708-5p was identified as a biomarker using this novel strategy. This study may provide some help for cancer marker screening.

PeerJ reviewing PDF | (2021:06:62872:1:1:NEW 1 Sep 2021) 


\section{Integrating microRNA expression, miRNA-mRNA}

\section{2 regulation network and signal pathway: a novel strategy for}

\section{Lung cancer biomarker discovery}

4

5 Renqing Nie, Wenling Niu, Tang Tang, Jin Zhang and Xiaoyi Zhang*

6

7 Faculty of Environment and Life, Beijing University of Technology, Beijing, China.

8

9 Corresponding Author:

10 Xiaoyi Zhang

11100 Pingleyuan, Beijing, 100124, China

12 Email address: zhangxiaoyi@bjut.edu.cn

13 


\section{ABSTRACT}

35 Background. Since there are inextricably connections among molecules in the biological networks, it would be a more efficient and accurate research strategy to screen microRNA (miRNA) markers combining with miRNA-mRNA regulatory networks. The independent regulation mode is more "fragile" and "influential" than the co-regulation mode. miRNAs can be used as biomarkers if they can independently regulate hub genes with important roles in the PPI network, simultaneously the expression products of the regulated hub genes play important roles in the signaling pathways of related tissue diseases.

Methods. We collected miRNA expression of non-small cell lung cancer (NSCLC) from The Cancer Genome Atlas (TCGA) database and the Gene Expression Omnibus (GEO) database. Volcano plot and signal-to-noise ratio (SNR) methods were used to obtain significant differentially expressed (SDE) miRNAs from the TCGA database and GEO database, respectively. A human miRNA-mRNA regulatory network was constructed and the number of genes uniquely targeted (NOG) by a certain miRNA was calculated. The Area Under the Curve (AUC) values were used to screen for clinical sensitivity and specificity. The candidate markers were obtained using the criteria of the top five maximum AUC values and NOG $\geq 3$. The protein-protein interaction (PPI) network was constructed and independently regulated hub genes were obtained. Gene Ontology (GO) analysis and KEGG pathway analysis were used to identify genes involved in cancer-related pathways. Finally, the miRNA which can independently regulate a hub gene and the hub gene can participate in an important cancer-related pathway was considered as a biomarker. The AUC values and gene expression profile analysis from two external GEO datasets as well as literature validation were used to verify the screening capability and reliability of this marker.

Results. 15 SDE miRNAs in lung cancer were obtained from the intersection of volcano plot and SNR based on the GEO database and the TCGA database. Five miRNAs with the top five maximum AUC values and NOG $\geq 3$ were screened out. 61 hub genes were obtained from the PPI network. It was found that the hub gene $G T F 2 F 2$ was independently regulated by miR-7085 p. Further pathway analysis indicated that $G T F 2 F 2$ participates in protein expression by binding with polymerase II, and it can regulate transcription and accelerate tumor growth. Hence, miR-708-5p could be used as a biomarker. The good screening capability and reliability of miR$708-5 \mathrm{p}$ as a lung cancer marker were confirmed by AUC values and gene expression profiling of external datasets, and experimental literature. The potential mechanism of miR-708-5p was proposed.

67 Conclusions. This study proposes a new idea for lung cancer marker screening by integrating microRNA expression, regulation network and signal pathway. miR-708-5p was identified as a 
69 biomarker using this novel strategy. This study may provide some help for cancer marker 70 screening.

\section{1. INTRODUCTION}

Lung cancer is the leading cause of cancer-related deaths worldwide (Siegel et al. 2017), and non-small cell lung cancer (NSCLC) accounts for the majority of new diagnoses. NSCLC is inherently incurable although many strategies have been proposed to improve patient survival (Fassina et al. 2011). Most patients are diagnosed with advanced disease, half of them with distant metastases at the time of initial diagnosis (Siegel et al. 2019), so patients often miss the best time for surgical and other treatments, resulting in a poor prognosis with a five-year survival rate of only $16 \%$ to $18 \%$ (Tanoue et al. 2015). However, if patients can be diagnosed early and receive treatment promptly, the five-year survival rate can be increased to $45 \%$ to $65 \%$ (Ettinger et al. 2010). Therefore, it has become increasingly important to detect lung cancer earlier.

The early diagnosis of lung cancer mainly relies on imaging, cytology and biochemical examination, but due to the limitation of the current treatment level, the false positive rate is very high (Xi et al. 2019). The gold standard of pathological examination is fiberoptic bronchoscopy and percutaneous lung biopsy (Diederich 2009), but these examinations have the disadvantages of invasiveness and poor compliance. Tumor marker tests, such as carcinoembryonic antigen (CEA), cytokeratin 19 fragment, squamous epithelial cell carcinoma (SCC) antigen, carcinoma antigen 125, neuron-specific enolase (NSE), have been widely used in the diagnosis of lung cancer, however, the sensitivity and specificity of these markers are not high (I \& Cho 2015). With the rapid development of high-throughput technologies, more and more biological data have been shown to be applicable to the prediction of cancer markers. Many studies have shown that miRNAs are closely correlated with the development of diseases, especially cancer (Elliot et al. 2019; Sun et al. 2018). The expression levels of miRNAs are statistically significantly different in the sera of patients with NSCLC compared to normal individuals (Liang et al. 2020; Ying et al. 2020), and nearly half of the annotated human miRNAs are located at vulnerable and critical points in the genome (Calin et al. 2004). miRNAs can exist stably without degradation in human tissues, blood and body fluids (Benz et al. 2016). They are small molecules and are specific in tissue expression and temporal expression, and can easily and accurately reflect the evolutionary pattern of disease development (Abrahamsson \& Dabrosin 2015), etc. Thus, miRNAs are promising markers for tumor diagnosis in clinical practice.

At present, most of the methods for identifying miRNA markers only consider the changes in the expression level of miRNA itself, and do not involve its regulatory genes. An individual biomolecule can't analyze the underlying mechanisms of various biological phenomena, but well-understood biological networks could lead to revealing the inherent laws of life activities at 
104 the systemic level (Menche et al. 2015). The differences in miRNA expression levels can be

105 reflected in the expression levels of the target genes regulated by miRNA (Bam et al. 2018),

106 therefore, the computational biology research method combined with miRNA-mRNA regulatory

107 networks would be a more efficient and accurate research strategy, and it can also provide new

108 ideas for finding cancer markers. Co-regulatory effects of miRNA-mRNA regulatory networks

109 are considered in some studies, but the results are somewhat influenced by the training set data

110 (Zhang \& Shen 2013). Zhang et al. (Zhang et al. 2014) proposed the independent regulation

111 ability of miRNA. Figure 1 shows the two types of regulation modes of miRNA-mRNA:

112 independent regulation mode means an mRNA is regulated by a unique miRNA, co-regulation

113 mode means an mRNA can be regulated by multiple miRNAs. The miRNA which can

114 independently regulate multiple mRNAs has a larger number of genes uniquely targeted (NOG)

115 value. Lin et al. (Lin et al. 2018a) reported that miRNAs with significant NOG in miRNA-

116 mRNA networks have stronger independent regulatory abilities, independent regulatory ability is

117 fragile and important for the stability of biological networks. The statistical evidence suggests

118 that most miRNAs that can be used as biomarkers have significantly larger NOG values, namely,

119 the independent regulatory ability would be an important network feature of miRNA biomarkers

120 (Lin et al. 2018b; Lin et al. 2016).

121 In addition, we believe that the regulatory ability of a miRNA also lies in the importance of

122 the genes regulated by the miRNA. The importance of the gene can be divided into two aspects:

123 one is whether the expression product of the gene is a core node in the protein-protein interaction

124 (PPI) network, highly connected hub gene plays an important role in the biological processes;

125 and the other is whether the expression product of the gene plays a role in an important signaling 126 pathway related to the disease.

127 Considering that the independent regulation mode of miRNA-mRNA is more "fragile", the

128 abnormal expression of the only miRNA will lead to a large change of this "fragile" structure, if

129 the expressed protein of the regulated gene act as a key node in the PPI network, the

130 dysregulation of the sole miRNA-gene interaction would result in a dramatic impact on

131 intracellular function, if this gene plays an essential role in a disease-related signaling pathway,

132 the disorder of independent regulation would inevitably lead to disease onset and progression.

133 This shows the huge influence of this independent regulation mode. Therefore, a new idea for

134 miRNA marker screening is proposed here: a hub gene can be independently regulated by a

135 significant differentially expressed (SDE) miRNA, and the express product of the hub gene plays

136 an important role in the signaling pathway of the relevant tissue disease, then the miRNA can be

137 used as a biomarker.

138 This study is going to use computational recognition to determine the SDE miRNAs

139 between lung cancer and paraneoplastic tissues. A regulatory network will be constructed and 
140 NOG value will be obtained. Then, the sensitivity and specificity of those SDE miRNAs would

141 be evaluated by Area Under the Curve (AUC) value. The miRNAs with the top five maximum

142 AUC values and NOG $\geq 3$ would be picked out as candidate markers. Then the importance of the

143 genes regulated by candidate markers was further investigated. Firstly, a PPI network will be

144 constructed using the target genes of these five candidate miRNAs, and the hub genes

145 independently regulated by candidate miRNA in the PPI network will be found out, and then

146 lung cancer-related signaling pathways participated by these hub genes would be analyzed. If

147 there is a hub gene that is independently regulated by a miRNA, and the expression product of

148 this gene plays an important role in lung cancer-related signaling pathways, then the miRNA can

149 be used as a biomarker. Finally, AUC values and gene expression profile analysis based on two

150 external GEO datasets as well as literature validation were used to verify the screening ability

151 and rationality of the marker. The potential mechanism of the biomarker will be assessed. The

152 workflow is displayed in Fig. 2.

153 2. MATERIALS \& METHODS

154 2.1 Data download and preprocessing

155 In order to eliminate the background difference of the obtained sample data, two different

156 databases were selected. miRNA-seq isoform quantification data was obtained from The Cancer

157 Genome Atlas (TCGA, https://portal.gdc.cancer.gov/) and GSE102286 dataset of the Gene

158 Expression Omnibus (GEO, https:/www.ncbi.nlm.nih.gov/geo/). The data from cancer tissues

159 and paraneoplastic tissue were paired up. Two datasets (GSE56036 and GSE36681) were

160 downloaded from the GEO as validation sets. The GSE56036 dataset contains 29 NSCLC

161 samples and 19 normal samples. We selected all 56 pairs of fresh frozen samples of NSCLC

162 tissue and normal tissue from the GSE36681 dataset.

163 The data was pre-processed. Firstly, the miRNAs with missing values of more than $20 \%$ in

164 all samples were removed. In additional, the Z-score method was used to find outliers and

165 replace the rest of missing values with medians. Due to the large gap between the number of

166 cancer samples and paraneoplastic samples, so smote algorithm was used to balance the data.

167 finally, the max-min normalization method was used to normalize the dataset.

\section{$168 \quad 2.2$ Obtaining candidate biomarkers}

\section{2.2.1 Computational identification of SDE miRNAs}

170 As a special scatter map, volcano plot was used to quickly identify those individuals who

171 have differences in the mass data, and it can intuitively display the data changes. There are two

172 important indexes of volcano plot, which are fold change (FC) and p-value (p-value). Threshold 
173 criteria of screening statistics: $|\log 2(\mathrm{FC})| \geq 1$, p-value $<0.05$. The results of the Volcano plot

174 were used for further screening by the signal-to-noise ratio (SNR) test (formula 1). The SNR

175 method is an effective method to remove noise, evaluate the classification ability of genes

176 according to the scoring level, discard the lower ranking genes and keep only the subsets with

177 high scores. It is a coarse-grained feature selection method.

$178 S N R=\frac{u_{+}(i)-u_{-}(i)}{\sigma_{+}(i)-\sigma_{-}(i)}$

179 In the above formula, $u_{+}(i)$ and $u_{-}(i)$ represent the average expression value of gene $i$ in normal 180 and tumor tissue samples, respectively. $\sigma_{+}(i)$ and $\sigma_{-}(i)$ represent the standard deviation of gene i in normal tissue samples and tumor tissue samples, respectively.

182 2.2.2 Construction of human miRNA-mRNA regulatory network and calculation of NOG

183

184

185

186

187

188

189

190

191

192

193

194

195

196

197

198

199

200

201

202

203

204

205

values

To obtain the independent regulatory capabilities (NOG value) of miRNA candidate markers, six commonly used public databases were used to construct human miRNA-mRNA regulatory networks. Among them, the data in the four databases of miR2Disease (http://www.mir2disease.org/7), miRecords (http://mirecords.biolead.org/), miRTarBase (http://mirtarbase.cuhk.edu.cn/php/index.php), and TarBase (http://www.microrna.gr/tarbase) mainly come from biological experiments such as high-throughput and low-throughput. The data of the other two databases, miRDB (http://mirdb.org) and miRWalk (http://mirwalk.umm.uniheidelberg.de), mainly come from computer algorithm prediction. The miRNA-mRNA regulatory network can be regarded as a unidirectional regulatory network. Some genes in this regulatory network are regulated only by a single miRNA, which means this miRNA has independent regulatory capabilities for this gene. If a miRNA regulates several genes independently, the number will be used as the NOG value of this miRNA. Generally, the NOG value of this miRNA is larger, the ability of the miRNA to independently regulate genes is stronger. In this study, the value of NOG $\geq 3$ indicates that the miRNA has strong independent regulatory capabilities, this criterion was used to screen candidate markers.

\subsubsection{Sensitivity and specificity analysis using ROC}

For SDE miRNAs, the ROC curve was plotted by the GraphPad Prism 8 software (http://www.graphpad.com) to identify the miRNAs which have good clinical sensitivity and specificity. The AUC can be used as a quantification standard for diagnostic classification capabilities. The larger the AUC value, the higher the diagnostic classification value. Criteria for screening diagnostic miRNA candidate markers: AUC $\geq 0.9$. Top 5 miRNAs were selected for follow-up research.

Peerj reviewing PDF | (2021:06:62872:1:1:NEW 1 Sep 2021) 


\section{$206 \quad 2.3$ Obtaining biomarkers}

207

208

209

210

211

212

213

214

215

216

217

218

219

220

221

222

223

224

225

226

227

228

229

230

231

232

233

234

235

236

237

\subsubsection{Construction of PPI network}

Target genes of candidate miRNAs were obtained using the constructed human miRNAmRNA network. Interactions between target gene products were constructed using the Search Tool for the Retrieval of Interacting Genes/Proteins (STRING; version 11.0) database and PPI networks were constructed using Cytoscape software (version 3.8.0; https://cytoscape.org/). The combined score (magnitude of the probability of protein interaction) should be greater than 0.9 (highest confidence). Nine methods of the CytoHubba - maximal clique centrality (MCC), the density of maximum neighborhood component (DMNC), maximum neighborhood component (MNC), degree, edge percolated component (EPC), closeness, radiality, betweenness and stress were used to calculate the score, and the upper quartiles of the scores were calculated separately. Genes with scores greater than the upper quartile for all nine methods were selected as hub genes.

\subsubsection{Bioinformatics analysis}

To gain insight into whether the expression products of those hub genes play a role in important disease-related signaling pathways, Gene Ontology (GO) analysis was performed using the DAVID (https://david.ncifcrf.gov/), and the online enrichment tool of KEGG (http://www.genome.jp/kegg/tool/map_pathway2.html) was used to perform the pathway analysis.

\subsubsection{Finding biomarkers}

A hub gene which is independently regulated by a miRNA, and the expression product of this gene plays an important role in lung cancer-related signaling pathways, then the miRNA can be used as a biomarker.

\subsection{Validation of the screening ability and reasonableness of biomarker}

\subsubsection{Reliability validated using expression profiling}

The differences in miRNA expression levels can be reflected in the expression levels of the target genes regulated by miRNA. So, the expression changes in lung cancer tissues of the marker miRNA and the hub gene independently regulated by it were verified. Expression data were downloaded from the TCGA database, and paired t-tests were performed on paired lung cancer and paraneoplastic samples using GraphPad Prism 8. Using Pearson correlation, we explored the association between miR-708-5p and GTF2F2 expression and plotted the correlation with R package ggstatsplot (https://github.com/IndrajeetPatil/ggstatsplot).

\subsubsection{Evaluating the performance of the marker miRNA using external datasets}


239

240

241

242

243

244

245

246

247

248

249

250

251

252

253

254

255

256

257

258

259

260

261

262

263

264

265

266

267

268

269

The GSE56036 and GSE36681 datasets were obtained from GEO database for the expression level evaluation of miR-708-5p. Then ROC analyses were performed using GraphPad Prism 8 to evaluate the diagnostic value of miR-708-5p.

\subsubsection{Reliability validated by Literatures}

The biomarkers were submitted to PubMed database. Literature screening criteria for a certain miRNA: "miR-XXX"[All Fields] AND "humans" [MeSH Terms]. Literature screening criteria for whether miRNA is associated with cancer:" Neoplasms"[Mesh] AND "miRXXX"[All Fields] AND "humans"[MeSH Terms]. Literature screening criteria for the association of amiRNA with lung cancer: "Lung Neoplasms"[Mesh] AND "miR-XXX"[All Fields] AND "humans"[MeSH Terms].

\section{RESULTS}

\subsection{Preprocessing results}

993 lung cancer tissue samples and 91 paraneoplastic tissue samples were downloaded from the TCGA database, each sample contains 2275 mature miRNAs. 59 lung cancer tissue samples and 59 paraneoplastic tissue samples were downloaded from the GEO database (GSE102286), each sample contains 654 mature miRNAs.

After sample balancing and three-step data preprocessing, there were 993 lung cancer tissue samples and 993 paraneoplastic tissue samples in the TCGA dataset, and the miRNAs in each tissue sample were reduced to 520. There were 59 lung cancer tissue samples and 59 paraneoplastic tissue samples, and the miRNAs in each sample were reduced to 125 in the GSE102286 dataset.

\subsection{Obtaining candidate biomarkers}

\subsubsection{Computational identification of SDE miRNAs}

Volcano plot result showed that 122 miRNAs in the TCGA database were up-regulated and 76 miRNAs were down-regulated, a total of 198 miRNAs with differential expression (Fig. 3A). The SNR result showed that 117 miRNAs with differential expression were screened from the 198 miRNAs (Fig. 3B; Table S1).

In the dataset GSE102286, the volcano plot results showed 29 miRNAs up-regulated and 10 down-regulated, totaling 39 miRNAs with differential expression (Fig. 3C). The SNR results showed that 29 miRNAs with differential expression were screened from the 39 miRNAs (Fig. 3D; Table S2). 
270

271

272

273

274

275

276

277

278

279

280

281

282

283

284

285

286

287

By using Venn diagrams, the outputs of the two databases were intersected as a set of miRNAs with significant differences. As shown in Fig. 3E, 15 SDE miRNAs (six up-regulated and nine down-regulated) were identified totally (Table 1)

\subsubsection{Results of human miRNA-mRNA regulatory network construction}

The human miRNA-mRNA regulatory network involves 1242 miRNAs, 14995 target genes, and 325240 action pairs. As shown in Fig. 4A, 554 (44.6\%) miRNAs in the regulatory network have independent regulatory capabilities, and 232 (18.7\%) miRNAs have strong independent regulatory capabilities $(\mathrm{NOG} \geq 3)$. In Fig. $4 \mathrm{~B}$, the distribution of the NOG values for miRNAs in the human miRNA-mRNA network followed a power-law distribution.

There are 10 miRNAs (miR-183-5p, miR-21-5p, miR-210-3p, miR-708-5p, miR-145-5p, miR-126-3p, miR-30a-5p, miR-218-5p, miR-96-5p and miR-1-3p) with NOG $\geq 3$ in 15 SDE miRNAs (Fig. 4C).

\subsubsection{Sensitivity and specificity analysis using ROC}

To further evaluate the clinical diagnostic sensitivity and specificity of 15 SDE miRNAs, the ROC curve was used. There are eight miRNAs with AUC $\geq 0.9$, they are miR-210-3p, miR1-3p, miR-96-5p, miR-183-5p, miR-708-5p, miR-144-3p, miR-218-5p and miR-30a-5p (Fig. 4D).

\subsubsection{Candidate biomarkers obtained from the intersection of NOD and ROC}

SDE miRNAs with top five AUC ranking (AUC $\geq 0.9$ ) and NOD $\geq 3$ were considered as miRNA candidate markers for lung cancer. Five candidate markers, namely, miR-210-3p, miR1-3p, miR-96-5p, miR-183-5p and miR-708-5p, were obtained. They have good diagnostic effects and also have strong independent regulation abilities.

\subsection{Obtaining biomarkers}

\subsubsection{PPI network construction and hub gene analysis}

The PPI network was constructed by selecting all the target genes of five miRNAs with a combined score $>0.9$ based on the STRING database. A node in the PPI network represents a protein, and the protein-protein interactions are presented by the link. The result is showed in Fig. 5 (Table S3).

The PPI network consisted of 1807 nodes and 15634 edges. A total of 61 hub genes were screened out (Table S4). Among these hub genes, GTF2F2 (purple circle in Fig. 5), independently regulated by miR-708-5p, has a high degree of connectivity, its degree is 83 (red circles and pink circles).

\subsubsection{Bioinformatics analysis}

Peer] reviewing PDF | (2021:06:62872:1:1:NEW 1 Sep 2021) 
303

304

305

306

307

308

309

310

311

312

313

314

315

316

317

318

319

320

321

322

323

324

325

326

327

328

329

330

331

332

333

334

335

336

To investigate whether the gene $G T F 2 F 2$ is involved in lung cancer-related pathways, functional analysis of $G T F 2 F 2$ was performed using DAVID and the results was shown in Fig. 6. The biological processes (BP) in which $G T F 2 F 2$ was involved were: transcriptional elongation from the RNA polymerase II promoter, regulation of transcription, DNA-templated, positive regulation of viral transcription. The main molecular functions (MF) were: transcription factor activity, core RNA polymerase II binding, ATP binding, protein binding. The cellular components (CC) were: nucleoplasm, nucleus, transcription factor TF2F complex.

KEGG (Kyoto Encyclopedia of Genes and Genomes) pathway of $G T F 2 F 2$ was analyzed, and the results showed that the target gene $G T F 2 F 2$ was involved in the pathway of hsa03022: Basal transcription factors. GTF $2 F 2$ binds with RNA polymerase II, and then participates in protein expression, which could regulate transcription and accelerate tumor growth (Kalkat et al. 2018).

\subsubsection{The final biomarkers}

In a word, we found that the target gene $G T F 2 F 2$, which was independently regulated by miR-708-5p, also existed as a key node in the PPI network, and it is in the pathway associated with cancer. According to our new idea for miRNA marker screening, miR-708-5p could be considered as a biomarker.

\subsection{Validation of the screening ability and reliability of the biomarker}

\subsubsection{Reliability validated using expression profiling}

GTF $2 F 2$ was independently regulated by miR-708-5p, and the expression level of miR-708$5 \mathrm{p}$ was significantly different, so the expression level of $G T F 2 F 2$ should have a significant difference also. To confirm this, the expression of miR-708-5P and GTF2F2 in paired lung tumor tissue samples and paraneoplastic tissue samples were downloaded from the TCGA database. The expression levels of miR-708-5p and GTF2F2 were significantly higher in lung tumor tissue samples than in paraneoplastic tissue samples (Figs. 7A and 7B; Tables S5 and S6). Pearson correlation presented that there was a significantly positive correlation between miR708-5p and GTF2F2 in Fig. 7C ( $\mathrm{r}=0.47, \mathrm{p}=1.63 \mathrm{e}-07)$.

\subsubsection{Evaluating the performance of the marker miRNA using external datasets}

Two external validation datasets were used to verify the performance of our marker miRNA. The expression level of miR-708-5p was significantly higher in NSCLC tissues than in normal tissues base on the GSE56036 and GSE36681 datasets (Figs. 8A and 8B; Tables S7 and S8). Additionally, the results of ROC analysis of the GSE56036 and GSE36681 datasets showed that miR-708-5p had good diagnostic values for NSCLC. The AUC of miR-708-5p was 0.835 in the GSE56036 dataset and 0.793 in the GSE36681dataset (Figs. 8C and 8D). 
337

338

339

340

341

342

343

344

345

346

347

348

349

350

351

352

353

354

355

356

357

358

359

360

361

362

363

364

365

366

367

368

369

370

371

\subsubsection{Reliability validated by literatures}

By searching and synthesizing the related literatures of miR-708-5p, we found that miR708-5p has been reported to be associated with lung cancer (Table 2).

Among the 10 retrieved papers related to lung cancer in Table 3, four papers did not state the relationship between miR-708-5p and lung cancer. One paper demonstrated their relationship with a bioinformatic approach, and in the rest five papers, the relationship between them was confirmed experimentally.

Using Immunoblotting, Dual-Luciferase reporter, and immunocytochemistry assays, Liu et al. (Liu et al. 2018) found that miR-708-5p could be used as a new diagnostic and prognostic marker for NSCLC, the study showed that miR-708-5p directly inhibited the translation of $D N M T 3 A$, resulting in a significant decrease in genome-wide DNA methylation and upregulation of the tumor suppressor $C D H 1$. The upregulation of $C D H 1$ reduced the activity of the Wnt/ $\beta$ catenin signaling pathway, which in turn affected the stem cell properties of NSCLC cells, and in clinical analysis, patients with increased miR-708-5p expression had significantly higher survival and lower recurrence rates. In the study by Xing et al. (Xing et al. 2010), miRNA expression was detected by GeneChip miRNA arrays in lung cancer tissue samples and RT-PCR in sputum, respectively, and all the results showed that miR-708-5p was highly expressed in lung cancer samples. Jang et al. (Jang et al. 2012) used microarray data analysis, $R T-q P C R$ and Luciferase reporter assay techniques to show that miR-708-5p was significantly more expressed in NSCLC tumor tissues than in paraneoplastic tissues. Wu et al. (Wu et al. 2016) used qRT-PCR and Luciferase assay showed that miR-708-5p expression inhibited lung cancer invasion and metastasis in vitro and in vivo. To define distinct molecular features of these two major histological subtypes of NSCLC, Molina-Pinelo, Sonia et al. (Molina-Pinelo et al. 2014) detected miR-708-5p overexpression in squamous cell lung cancer compared to adenocarcinoma by TaqMan low-density array and qPCR methods.

These reports experimentally confirmed that miR-708-5p is highly expressed in lung cancer, containing NSCLC, can be used as a diagnostic and prognostic marker. Our finding is consistent with these experimental results.

\section{DISCUSSION}

How does miR-708-5p play a role in lung carcinogenesis and development through independent regulation of the hub gene $G T F 2 F 2$ ? A detailed analysis of its potential regulatory mechanism was explored here.

The general transcription factor complex GTF2 A, B, E, F and $\mathrm{H}$ were jointly involved in protein expression (Flores et al. 1990). Among them, $G T F 2 F$ was a tetrameric molecule consisting of two subunits, GTF2F1 (RAP74) and GTF2F2 (RAP30) (Thomas \& Chiang 2006). 
372 Human $G T F 2 F$ was widely expressed in various tissues and organs of the body, especially in the

373 liver and lung. GTF2F2 was involved in gene transcription initiation, promoter clearance, and

374 elongation, and it was linked to $G T F 2 F 1$ via the N-terminus. The middle part of GTF2F2 can

375 bind to the 5th subunit of RNA polymerase II (Pol II), RPB5, through RPB5-mediating protein

376 (RMP), hence helping Pol II to bind to the promoter region and influence subsequent

377 transcriptional elongation (Jentsch et al. 2002). At the C-terminus of GTF2F2, there was also a

378 non-specific DNA-binding structural domain that possessed a characteristic ATP-dependent

379 DNA unwinding enzyme activity. GTF2F2 as a target molecule for many important transcription

380 factors affected the formation or stability of the transcription initiation complex, thus influencing

381 and regulating the transcription process.

382 It has been established that changes in $G T F 2 F 2$ function will lead to serious diseases

383 (Bansard et al. 2011; Kaplan \& Stockwell 2012). However, the mechanism of GTF2F with

384 cancer is not clear. The MYC protein family played an important role in normal physiology,

385 proliferation and development, and its dysregulated expression in cancer was associated with

386 poor prognosis and disease aggression (Kalkat et al. 2017). MYC functions as a master

387 regulatory transcription factor that binds to and regulates the expression of thousands of target

388 genes (Cole \& McMahon 1999). The MYC family encoded transcription factors containing six

389 highly conserved regions, termed MYC homology boxes (MBs), among them, MB0 directly

390 interacts with TFIIF in a transcription elongation complex, containing CDK9 and Pol II, MB0 is

391 dispensable for tumor initiation but is a major accelerator of tumor growth (Kalkat et al. 2018).

392 HBx was found to target RPB5 to stimulate transactivation (Cheong et al. 1995), RPB5-

393 mediating protein (RMP) interacted with RPB5 and counteracts transactivation by HBx. TF2F2

394 has been shown to associate with pol II and recruit pol II to the promoter in the transcription

395 initiation (Garrett et al. 1992; Roeder 1996; Sopta et al. 1989; Wei et al. 2001). TF2F1 binds to

396 the initiation complex will allow pol II to make promoter contact (Joliot et al. 1995; Zhu et al.

397 1994). The D5 region of RMP is necessary and sufficient for the association between RMP and

398 GTF2F. Interaction with GTF2F is required for the suppression of activated transcription by

399 RMP. RMP regulates the genes related to apoptosis and cell cycle, plays an antiapoptotic role in

400 the proliferation and growth of HCC cells. BCL2, a gene that inhibits apoptosis, was elevated in

401 overexpressing RMP (RMPo) tumors, while $B A X$, a gene that drives apoptosis, was decreased in

402 RMPo tumors. Meanwhile, depletion of RMP induced G2 arrest in HCC cells by reducing the

403 expression of Cdk1 and Cyclin B (Yang et al. 2011). From the above studies, we can conclude

404 that TF2F can interact with different factors to influence the cell carcinogenesis process, for

405 example, it binds to MB0 of MYC to promote cancer development, and binds to RMP to further

406 inhibit the expression of pro-apoptotic genes and promote the expression of cell cycle-related

407 genes to promote malignant tumorigenesis. It can be seen that if the expression of TF2F is

Peer) reviewing PDF | (2021:06:62872:1:1:NEW 1 Sep 2021) 
408 regulated, it must be closely related to cell canceration. The schematic diagram of $G T F 2 F 2$ 409 related functions is shown in Fig. 9.

410 It has also been demonstrated that miR-708-5p plays an important regulatory role in the 411 development of lung cancer. Jang et al. (Jang et al. 2012) reported that overexpression of miR-

$412708-5 p$ increased proliferation and invasion of lung cancer cells by experimental methods, and

413 further identified a valuable link that miR-708-5p may directly downregulate TMEM 88 that

414 weakened Wnt activity to promote lung cancer progression. In the contrary, the work of

415 Monteleone et al. (Monteleone \& Lutz 2020) demonstrated that miR-708-5P exerted an

416 inhibitory effect on lung carcinogenesis by suppressing Prostaglandin E2 (PGE2) signaling.

417 Their work evidenced that miR-708-5p directly downregulated Cyclooxygenase-2 (COX-2) and

418 microsomal prostaglandin E synthase 1 (mPGES-1). COX-2 and mPGES-1 promoted PGE2

419 synthesis in the arachidonic acid metabolism pathway.

420 Our results also showed that miR-708-5p had an important role in lung carcinogenesis and 421 development, which was consistent with the results of existing studies, but the pathway of action 422 was different from the existing studies. Our study found that in lung tissues, the gene of TF2F2 423 was independently regulated by miR-708-5p, and the gene of TF2F2 belonged to the core gene. 424 As a core gene, the expression product of the TF2F2 gene was very important in the protein 425 interaction network. At the same time, the regulation of this gene was very sensitive and fragile, 426 and in lung tissue, it was only independently regulated by miR-708-5p. If the expression of miR$427708-5 p$ changes, the protein interaction network will be greatly affected, and further cellular 428 functions will be greatly affected. In-depth analysis revealed that TF2F can interact with 429 different factors to influence the cell carcinogenesis process, for example, it binds to MB0 of 430 MYC to promote cancer development and binds to RMP to further inhibit the expression of pro431 apoptotic genes and promote the expression of cell cycle-related genes to promote malignant 432 tumorigenesis. The above mechanism has not been reported so far.

433 The limitation of this study should be considered. Since the strategy in this study was only 434 used to identify non-small cell lung cancer miRNA markers and was not widely applied to the 435 identification of other disease markers, the generalizability of the strategy needs to be further 436 evaluated. Because network regulation in the human body is very complex, it needs to be 437 evaluated with more extensive disease and data to see whether the role of vulnerable nodes in 438 biological networks can be influenced and weakened by other co-regulatory networks.

\section{CONCLUSIONS}

This study proposed a novel idea for marker screening, that is, miRNAs can be used as biomarkers if they can independently regulate hub genes with important roles in the PPI network, 
442 and the expression products of the regulated hub genes play important roles in the signaling 443 pathways of related tissue diseases.

444 In this study, for lung cancer, 15 SDE miRNAs were identified, and the SDE miRNAs with 445 the top five AUC values and NOG $\geq 3$ were selected as candidate markers, they were considered 446 to have good sensitivity and specificity, and also have strong independent regulation abilities.

447 Then, the importance of the target gene of candidate markers was investigated. The PPI network 448 was constructed and biological function and pathway analysis were performed. The highly 449 connected hub gene plays an important role in the biological processes, and generally, is an 450 important target. 61 hub genes regulated by these five candidate miRNAs were obtained. We 451 found the important hub gene $G T F 2 F 2$, which was independently regulated by miR-708-5p, and 452 it interacted with RNA Pol II as a basal transcription factor to perform transcriptional functions 453 and accelerate tumor growth. In a word, the gene GTF2F2 has an important role in the induction 454 and progression of lung cancer, at the same time, it is subject to a fragile independent regulation. 455 Dysregulation of the fragile sole miRNA-gene interaction would inevitably lead to the 456 occurrence and development of diseases. Therefore, miR-708-5p, the regulator of this gene, can 457 be considered as a biomarker. Finally, the screening ability of miR-708-5p was evaluated by the 458 AUC values of two external GEO datasets. The reliability of miR-708-5p was confirmed by 459 expression level change of miR-708-5p and its target gene GTF2F2 and by experimentally 460 literature validation.

461 This study further explored the potential mechanism of miR-708-5p in lung cancer tissue.

$462 \mathrm{miR}-708-5 \mathrm{p}$ plays a role in lung carcinogenesis and development through independent regulation 463 of the hub gene $G T F 2 F 2$. the disorder of the independent regulation is easy to cause dramatic 464 changes in intracellular function and cause lung carcinogenesis and development. This 465 mechanism has not been reported.

466 6. ACKNOWLEDGMENTS

467 Thanks to all the researchers and staff working for The Cancer Genome Atlas database and Gene 468 Expression Omnibus database.

469

470

471

472

473

474

475

476

\section{References}

Abrahamsson A, and Dabrosin C. 2015. Tissue specific expression of extracellular microRNA in human breast cancers and normal human breast tissue in vivo. Oncotarget 6:22959-22969.

Bam M, Yang X, Sen S, Zumbrun EE, Dennis L, Zhang J, Nagarkatti PS, and Nagarkatti M. 2018. Characterization of Dysregulated miRNA in Peripheral Blood Mononuclear Cells from Ischemic Stroke Patients. Mol Neurobiol 55:1419-1429. $10.1007 / \mathrm{s} 12035-016-0347-8$ 
477

478

479

480

481

482

483

484

485

486

487

488

489

490

491

492

493

494

495

496

497

498

499

500

501

502

503

504

505

506

507

508

509

510

511

512

513

514

515

516

Bansard C, Lequerré T, Derambure C, Vittecoq O, Hiron M, Daragon A, Pouplin S, Daveau M, Boyer O, Tron F, Le Loët X, and Salier J-P. 2011. Gene profiling predicts rheumatoid arthritis responsiveness to IL-1Ra (anakinra). Rheumatology (Oxford, England) 50:283-292. 10.1093/rheumatology/keq344

Benz F, Roy S, Trautwein C, Roderburg C, and Luedde T. 2016. Circulating MicroRNAs as Biomarkers for Sepsis. Int J Mol Sci 17. 10.3390/ijms 17010078

Calin GA, Sevignani C, Dumitru CD, Hyslop T, Noch E, Yendamuri S, Shimizu M, Rattan S, Bullrich F, Negrini M, and Croce CM. 2004. Human microRNA genes are frequently located at fragile sites and genomic regions involved in cancers. Proceedings of the National Academy of Sciences of the United States of America 101:2999-3004.

Cheong JH, Yi M, Lin Y, and Murakami S. 1995. Human RPB5, a subunit shared by eukaryotic nuclear RNA polymerases, binds human hepatitis B virus X protein and may play a role in X transactivation. The EMBO journal 14:143-150.

Cole MD, and McMahon SB. 1999. The Myc oncoprotein: a critical evaluation of transactivation and target gene regulation. Oncogene 18:2916-2924.

Diederich S. 2009. Pulmonary nodules: do we need a separate algorithm for nonsolid lesions? Cancer imaging : the official publication of the International Cancer Imaging Society 9 Spec No A:S126-S128. 10.1102/1470-7330.2009.9046

Elliot S, Periera-Simon S, Xia X, Catanuto P, Rubio G, Shahzeidi S, El Salem F, Shapiro J, Briegel K, Korach KS, and Glassberg MK. 2019. MicroRNA let-7 Downregulates Ligand-Independent Estrogen Receptor-mediated Male-Predominant Pulmonary Fibrosis. American journal of respiratory and critical care medicine 200:1246-1257. 10.1164/rccm.201903-0508OC

Ettinger DS, Akerley W, Bepler G, Blum MG, Chang A, Cheney RT, Chirieac LR, D'Amico TA, Demmy TL, Ganti AKP, Govindan R, Grannis FW, Jahan T, Jahanzeb M, Johnson DH, Kessinger A, Komaki R, Kong F-M, Kris MG, Krug LM, Le Q-T, Lennes IT, Martins R, O'Malley J, Osarogiagbon RU, Otterson GA, Patel JD, Pisters KM, Reckamp K, Riely GJ, Rohren E, Simon GR, Swanson SJ, Wood DE, and Yang SC. 2010. Non-small cell lung cancer. Journal of the National Comprehensive Cancer Network: JNCCN 8:740-801.

Fassina A, Cappellesso R, and Fassan M. 2011. Classification of non-small cell lung carcinoma in transthoracic needle specimens using microRNA expression profiling. Chest 140:1305-1311. 10.1378/chest.11-0708

Flores O, Ha I, and Reinberg D. 1990. Factors involved in specific transcription by mammalian RNA polymerase II. Purification and subunit composition of transcription factor IIF. The Journal of biological chemistry 265:5629-5634.

Garrett KP, Serizawa H, Hanley JP, Bradsher JN, Tsuboi A, Arai N, Yokota T, Arai $\mathrm{K}$, Conaway RC, and Conaway JW. 1992. The carboxyl terminus of RAP30 is similar in sequence to region 4 of bacterial sigma factors and is required for function. The Journal of biological chemistry 267:23942-23949. 
517

518

519

520

521

522

523

524

525

526

527

528

529

530

531

532

533

534

535

536

537

538

539

540

541

542

543

544

545

546

547

548

549

550

551

552

553

554

555
I H, and Cho J-Y. 2015. Lung Cancer Biomarkers. Advances in clinical chemistry 72:107-170. 10.1016/bs.acc.2015.07.003

Jang JS, Jeon H-S, Sun Z, Aubry MC, Tang H, Park C-H, Rakhshan F, Schultz DA, Kolbert CP, Lupu R, Park JY, Harris CC, Yang P, and Jen J. 2012. Increased miR-708 expression in NSCLC and its association with poor survival in lung adenocarcinoma from never smokers. Clinical cancer research : an official journal of the American Association for Cancer Research 18:3658-3667. 10.1158/1078-0432.CCR-11-2857

Jentsch TJ, Stein V, Weinreich F, and Zdebik AA. 2002. Molecular structure and physiological function of chloride channels. Physiological reviews 82:503-568.

Joliot V, Demma M, and Prywes R. 1995. Interaction with RAP74 subunit of TFIIF is required for transcriptional activation by serum response factor. Nature 373:632-635.

Kalkat M, De Melo J, Hickman KA, Lourenco C, Redel C, Resetca D, Tamachi A, Tu WB, and Penn LZ. 2017. MYC Deregulation in Primary Human Cancers. Genes 8. 10.3390/genes8060151

Kalkat M, Resetca D, Lourenco C, Chan P-K, Wei Y, Shiah Y-J, Vitkin N, Tong Y, Sunnerhagen M, Done SJ, Boutros PC, Raught B, and Penn LZ. 2018. MYC Protein Interactome Profiling Reveals Functionally Distinct Regions that Cooperate to Drive Tumorigenesis. Molecular cell 72. 10.1016/j.molcel.2018.09.031

Kaplan A, and Stockwell BR. 2012. Therapeutic approaches to preventing cell death in Huntington disease. Progress in neurobiology 99:262-280.

10.1016/j.pneurobio.2012.08.004

Liang G, Meng W, Huang X, Zhu W, Yin C, Wang C, Fassan M, Yu Y, Kudo M, Xiao S, Zhao C, Zou P, Wang Y, Li X, Croce CM, and Cui R. 2020. miR-196b-5pmediated downregulation of TSPAN12 and GATA6 promotes tumor progression in nonsmall cell lung cancer. Proceedings of the National Academy of Sciences of the United States of America 117:4347-4357. 10.1073/pnas.1917531117

Lin Y, Chen F, Shen L, Tang X, Du C, Sun Z, Ding H, Chen J, and Shen B. 2018a. Biomarker microRNAs for prostate cancer metastasis: screened with a network vulnerability analysis model. Journal of translational medicine 16:134. 10.1186/s12967018-1506-7

Lin Y, Wu W, Sun Z, Shen L, and Shen B. 2018b. MiRNA-BD: an evidence-based bioinformatics model and software tool for microRNA biomarker discovery. $R N A$ biology 15:1093-1105. 10.1080/15476286.2018.1502590

Lin Y, Yuan X, and Shen B. 2016. Network-Based Biomedical Data Analysis. Advances in experimental medicine and biology 939:309-332.

Liu T, Wu X, Chen T, Luo Z, and Hu X. 2018. Downregulation of DNMT3A by miR-708-5p Inhibits Lung Cancer Stem Cell-like Phenotypes through Repressing Wnt/ $\beta$ catenin Signaling. Clinical cancer research : an official journal of the American Association for Cancer Research 24:1748-1760. 10.1158/1078-0432.CCR-17-1169 
556

557

558

559

560

561

562

563

564

565

566

567

568

569

570

571

572

573

574

575

576

577

578

579

580

581

582

583

584

585

586

587

588

589

590

591

592

593

594

595

596
Menche J, Sharma A, Kitsak M, Ghiassian SD, Vidal M, Loscalzo J, and Barabasi AL. 2015. Disease networks. Uncovering disease-disease relationships through the incomplete interactome. Science 347:1257601. 10.1126/science.1257601

Molina-Pinelo S, Gutiérrez G, Pastor MD, Hergueta M, Moreno-Bueno G, GarcíaCarbonero R, Nogal A, Suárez R, Salinas A, Pozo-Rodríguez F, Lopez-Rios F, AgullóOrtuño MT, Ferrer I, Perpiñá A, Palacios J, Carnero A, and Paz-Ares L. 2014. MicroRNA-dependent regulation of transcription in non-small cell lung cancer. PloS one 9:e90524. 10.1371/journal.pone.0090524

Monteleone NJ, and Lutz CS. 2020. miR-708-5p targets oncogenic prostaglandin E2 production to suppress a pro-tumorigenic phenotype in lung cancer cells. Oncotarget 11:2464-2483. 10.18632/oncotarget.27614

Roeder RG. 1996. The role of general initiation factors in transcription by RNA polymerase II. Trends in biochemical sciences 21:327-335.

Siegel RL, Miller KD, and Jemal A. 2017. Cancer Statistics, 2017. CA Cancer J Clin 67:7-30. 10.3322/caac.21387

Siegel RL, Miller KD, and Jemal A. 2019. Cancer statistics, 2019. CA Cancer J Clin 69:7-34. 10.3322/caac.21551

Sopta M, Burton ZF, and Greenblatt J. 1989. Structure and associated DNA-helicase activity of a general transcription initiation factor that binds to RNA polymerase II. Nature 341:410-414.

Sun Z, Shi K, Yang S, Liu J, Zhou Q, Wang G, Song J, Li Z, Zhang Z, and Yuan W. 2018. Effect of exosomal miRNA on cancer biology and clinical applications. Mol Cancer 17:147. 10.1186/s12943-018-0897-7

Tanoue LT, Tanner NT, Gould MK, and Silvestri GA. 2015. Lung cancer screening. American journal of respiratory and critical care medicine 191:19-33.

10.1164/rccm.201410-1777CI

Thomas MC, and Chiang C-M. 2006. The general transcription machinery and general cofactors. Critical reviews in biochemistry and molecular biology 41:105-178.

Wei W, Dorjsuren D, Lin Y, Qin W, Nomura T, Hayashi N, and Murakami S. 2001. Direct interaction between the subunit RAP30 of transcription factor IIF (TFIIF) and RNA polymerase subunit 5, which contributes to the association between TFIIF and RNA polymerase II. The Journal of biological chemistry 276:12266-12273.

Wu X, Liu T, Fang O, Dong W, Zhang F, Leach L, Hu X, and Luo Z. 2016. MicroRNA-708-5p acts as a therapeutic agent against metastatic lung cancer. Oncotarget 7:2417-2432. 10.18632/oncotarget.6594

Xi K, Wang W, Wen Y, Chen Y, Zhang X, Wu Y, Zhang R, Wang G, Huang Z, and Zhang L. 2019. Combining Plasma miRNAs and Computed Tomography Features to Differentiate the Nature of Pulmonary Nodules. Front Oncol 9:975.

10.3389/fonc.2019.00975

Xing L, Todd NW, Yu L, Fang H, and Jiang F. 2010. Early detection of squamous cell lung cancer in sputum by a panel of microRNA markers. Modern pathology : an 
597

598

599

600

601

602

603

604

605

606

607

608

609

610

611

612

613

614

615

616

617

618 official journal of the United States and Canadian Academy of Pathology, Inc 23:11571164. 10.1038/modpathol.2010.111

Yang H, Gu J, Zheng Q, Li M, Lian X, Miao J, Jiang J, and Wei W. 2011. RPB5mediating protein is required for the proliferation of hepatocellular carcinoma cells. The Journal of biological chemistry 286:11865-11874. 10.1074/jbc.M110.136929

Ying L, Du L, Zou R, Shi L, Zhang N, Jin J, Xu C, Zhang F, Zhu C, Wu J, Chen K, Huang M, Wu Y, Zhang Y, Zheng W, Pan X, Chen B, Lin A, Tam JKC, van Dam RM, Lai DTM, Chia KS, Zhou L, Too HP, Yu H, Mao W, and Su D. 2020. Development of a serum miRNA panel for detection of early stage non-small cell lung cancer. Proceedings of the National Academy of Sciences of the United States of America 117:25036-25042. 10.1073/pnas.2006212117

Zhang W, and Shen B. 2013. Identification of Cancer MicroRNA Biomarkers Based on miRNA-mRNA Network. In: Shen B, ed. Bioinformatics for Diagnosis, Prognosis and Treatment of Complex Diseases. Dordrecht: Springer Netherlands, 153-167.

Zhang W, Zang J, Jing X, Sun Z, Yan W, Yang D, Shen B, and Guo F. 2014. Identification of candidate miRNA biomarkers from miRNA regulatory network with application to prostate cancer. Journal of translational medicine 12:66. 10.1186/14795876-12-66

Zhu H, Joliot V, and Prywes R. 1994. Role of transcription factor TFIIF in serum response factor-activated transcription. The Journal of biological chemistry 269:34893497. 
Figure 1

Two regulatory types of miRNAs in the human miRNA-mRNA network.

The red circles represent mRNAs regulated by a single miRNA, the grey circles represent mRNAs regulated by multiple miRNAs. NOG, the number of genes uniquely targeted.

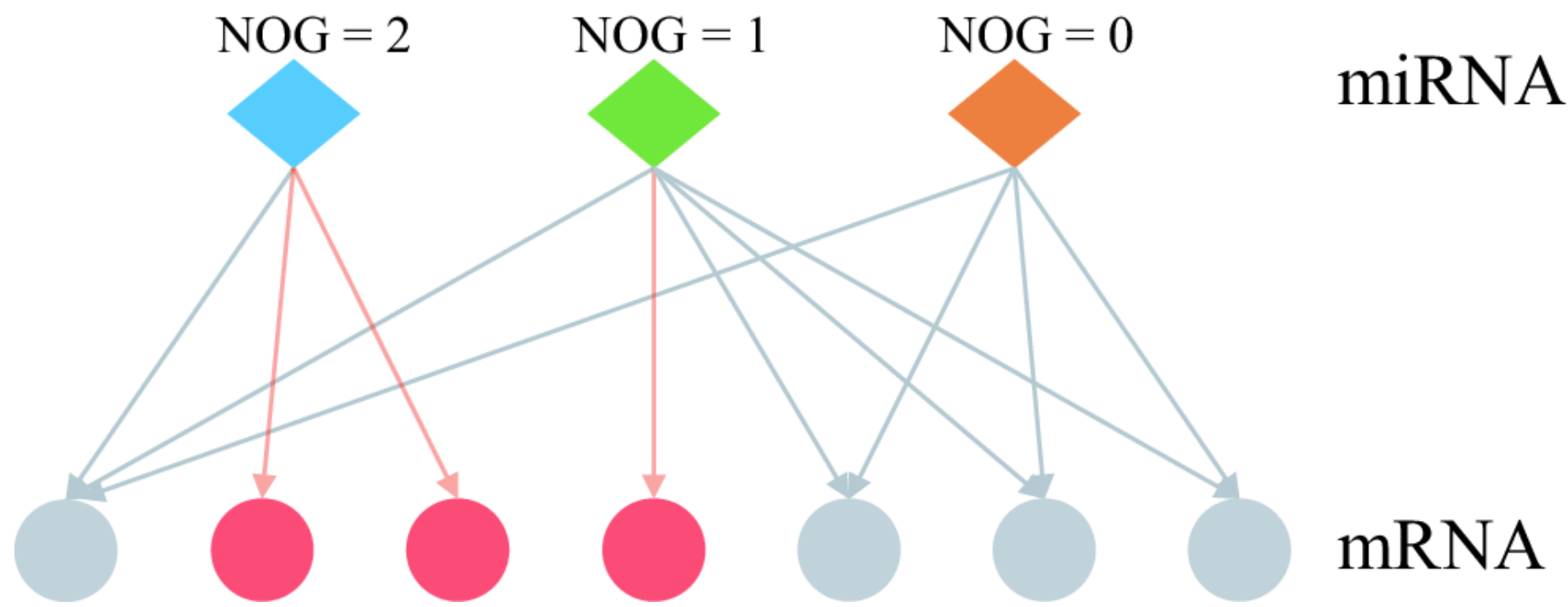


Figure 2

The workflow of this study.

SDE, significant differentially expressed; NOG, the number of genes uniquely targeted.

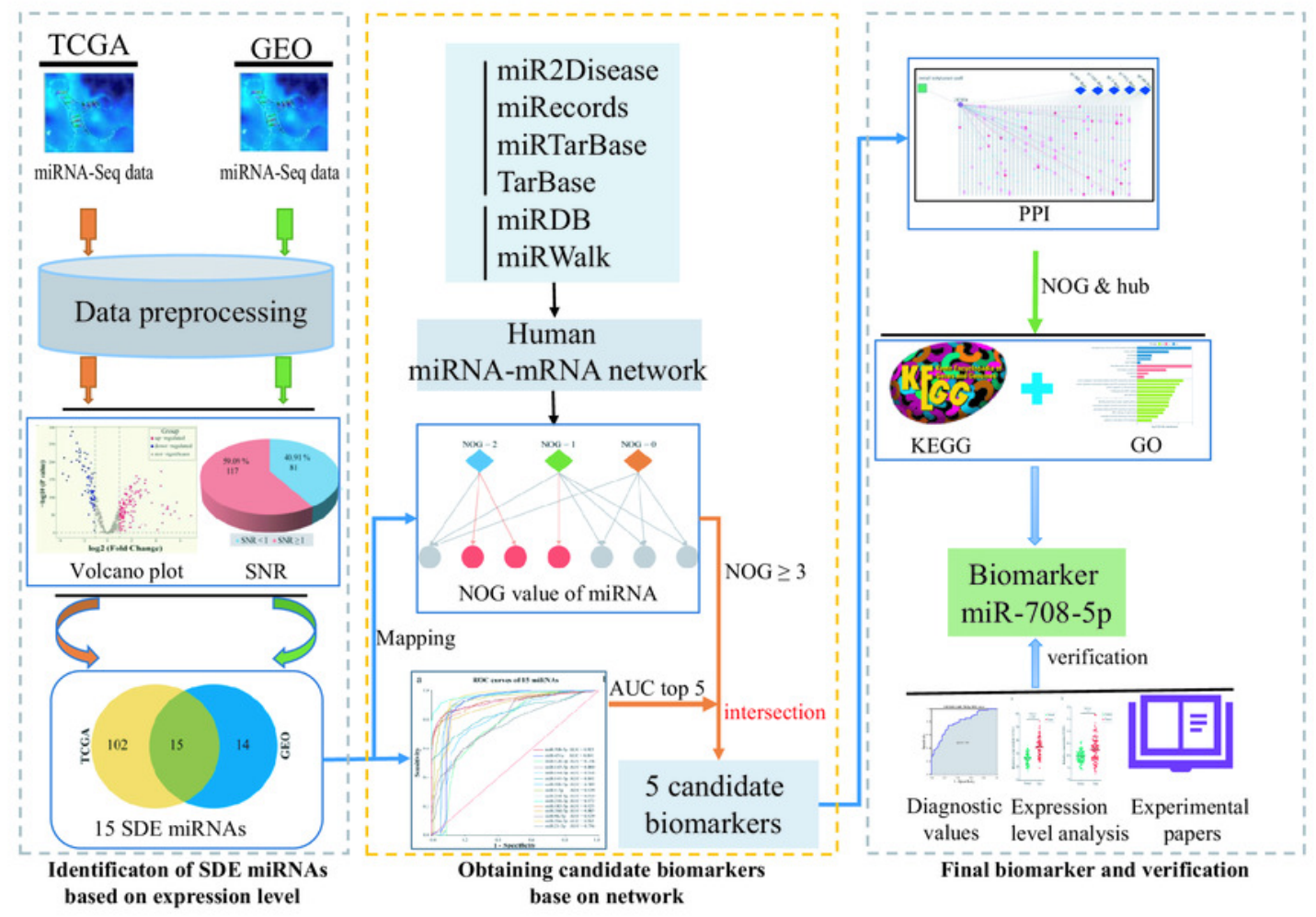




\section{Figure 3}

Results of SDE miRNAs.

Volcano (A) and signal-to-noise ratio (B) from TCGA, volcano (C) and signal-to-noise ratio (D) from GEO, A and C, significantly down-regulated and up-regulated miRNAs are painted in blue and red. Venn diagram (E) showed the overlapping results of SDE miRNAs from TCGA and GEO.

A

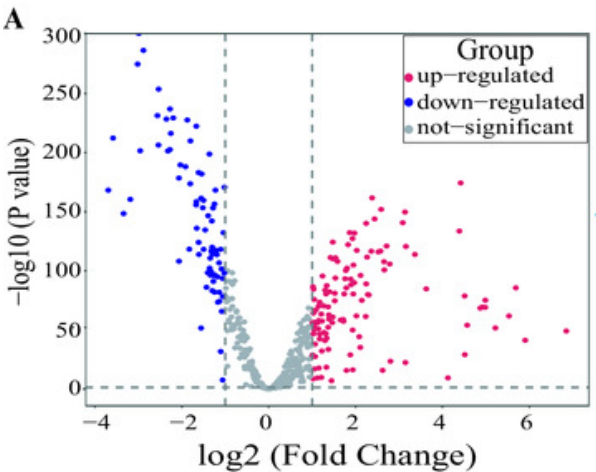

B

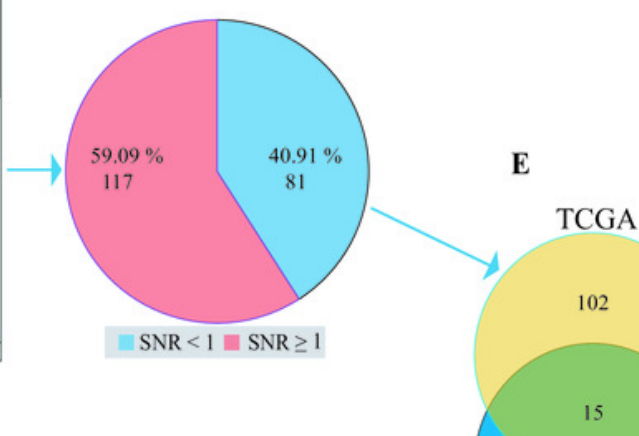

C

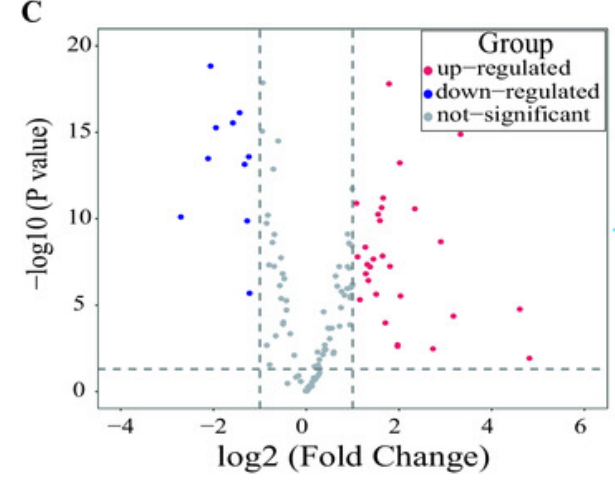

D

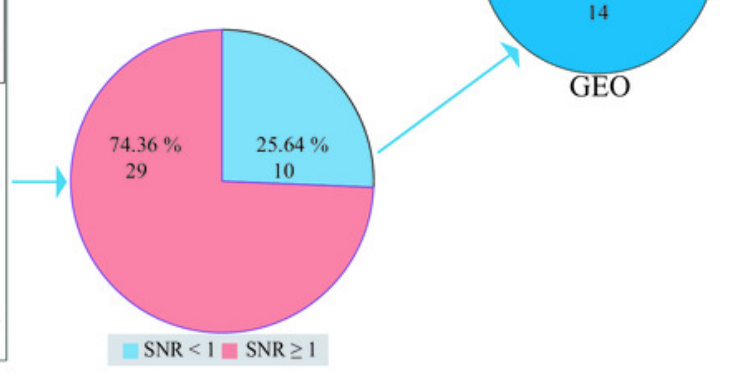


Figure 4

NOG statistics of miRNAs in human miRNA-mRNA network and ROC curves of SDE miRNAs.

$(A, B)$ the NOG distribution of miRNAs in the human miRNA-mRNA regulatory network. $(C)$

NOG values of 15 SDE miRNAs. (D) ROC curves for 15 SDE miRNAs were plotted based on the TCGA data. 
A

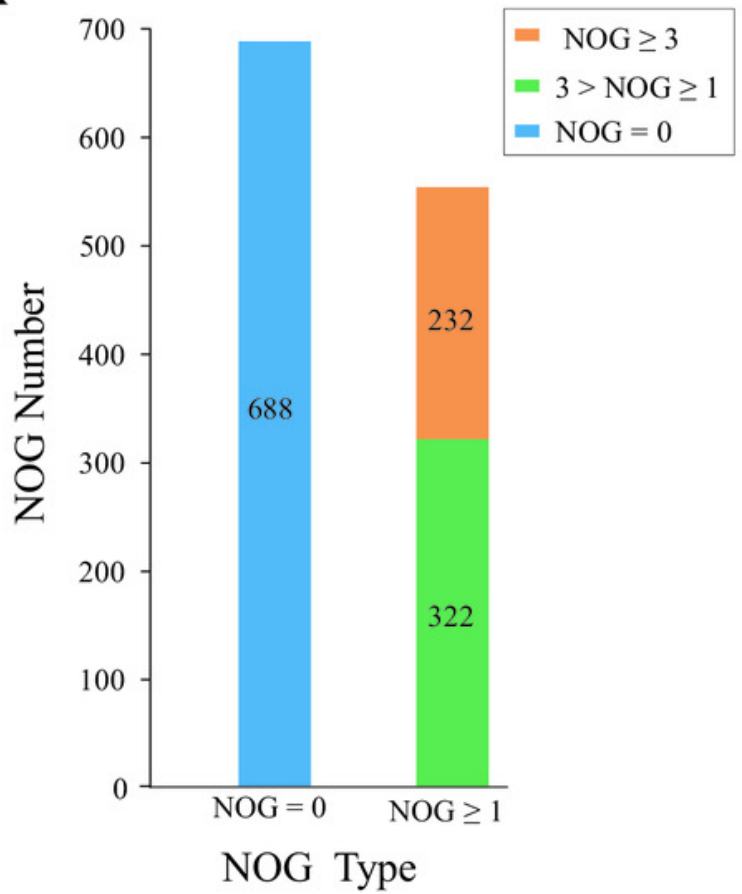

B

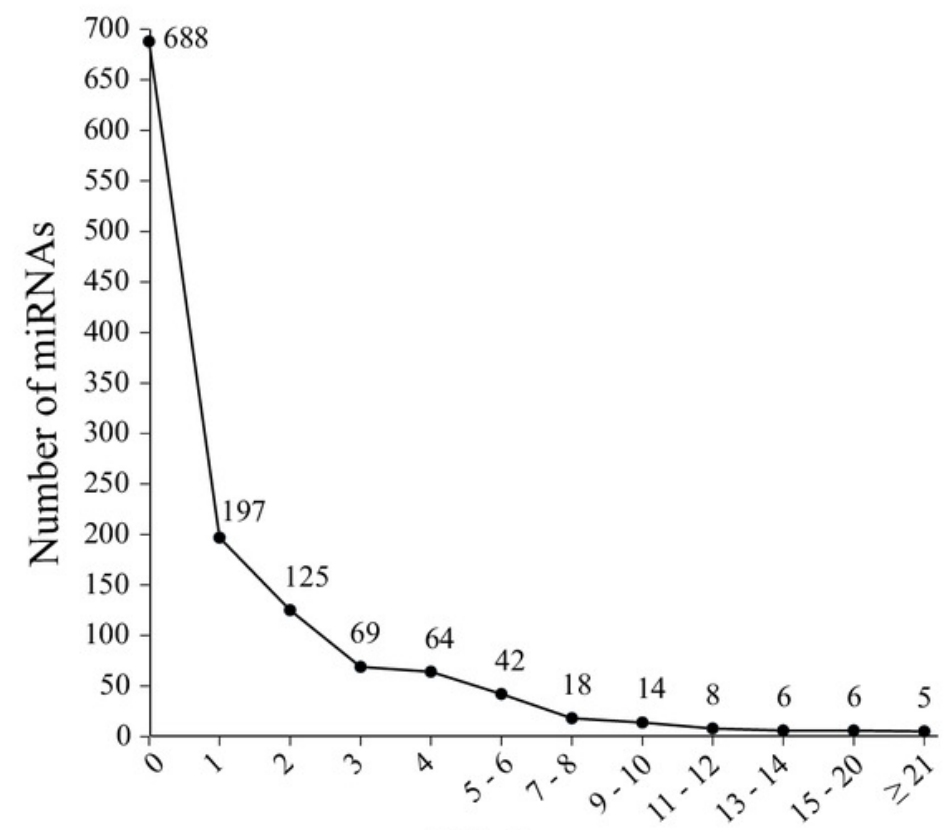

NOG range

D

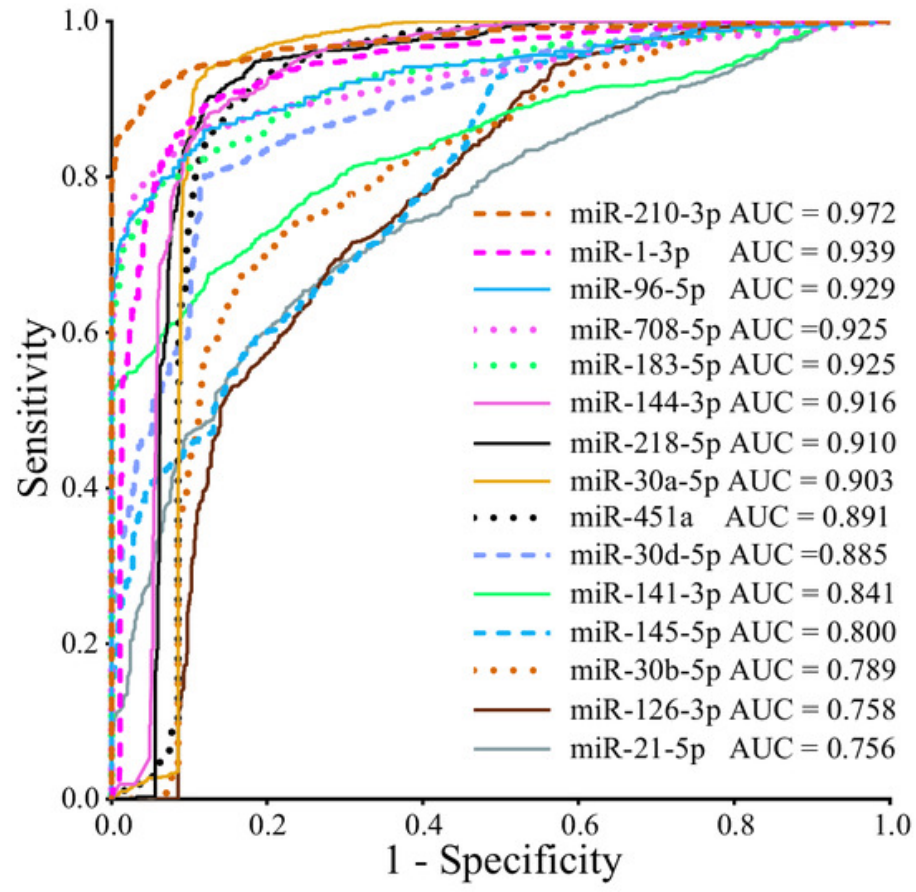




\section{Figure 5}

PPI of five miRNA target genes.

The diamonds represent miRNAs, the circles stand for genes, and the square stands for a pathway. The rectangular area in the diagram is the PPI network. Purple circle, red circles and blue circles are hub genes. The purple circle (GTF2F2) is both hub gene and gene independently regulated by miR-708-5P. The red circles represent genes that are both hub genes and interact with GTF2F2. The pink circles represent genes that interact with GTF2F2 but are not hub genes.

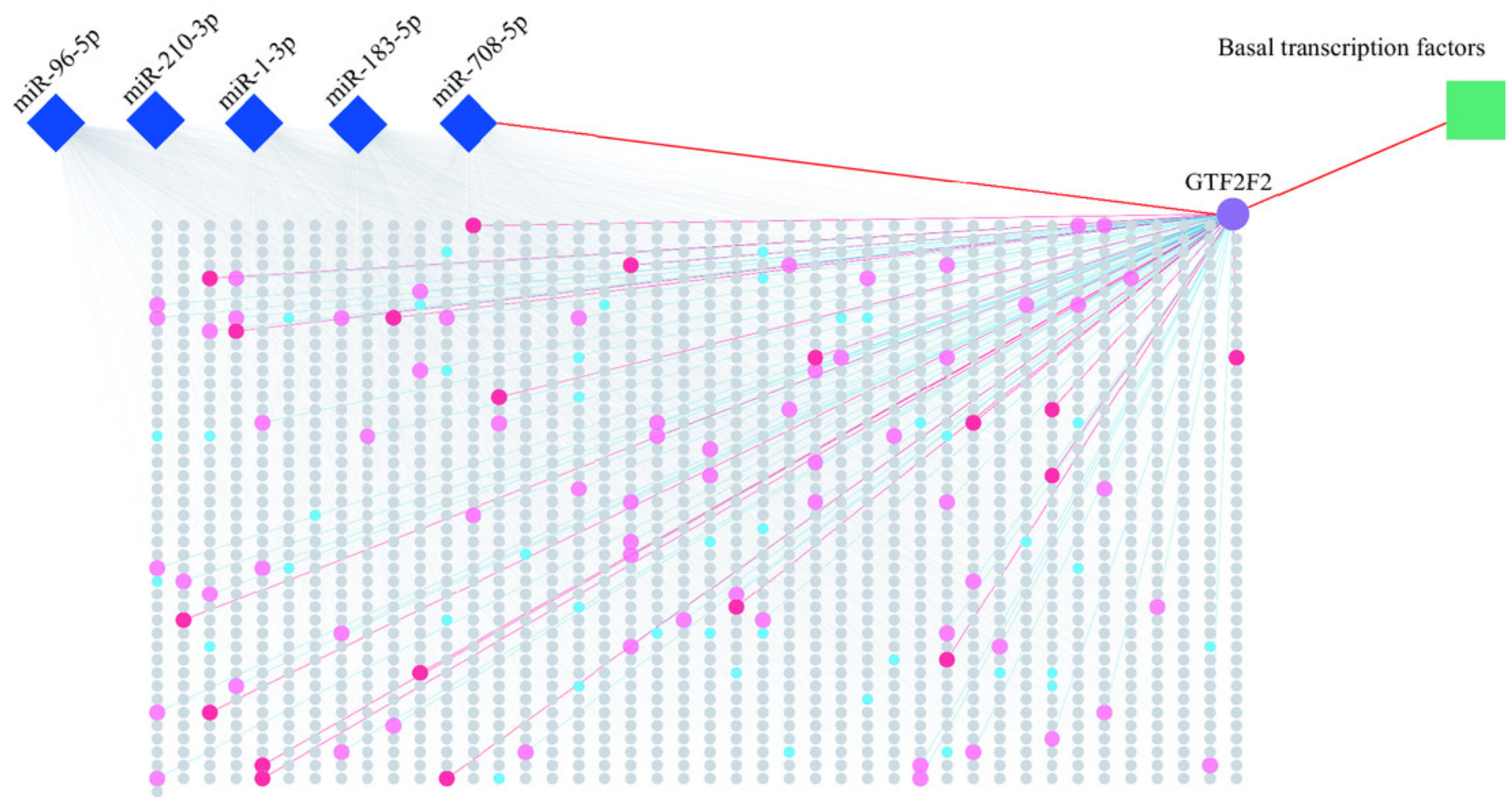




\section{Figure 6}

\section{GO functional analysis of GTF2F2.}

\section{BP: Biological Process. MF, Molecular Function. CC, Cellular Component.}

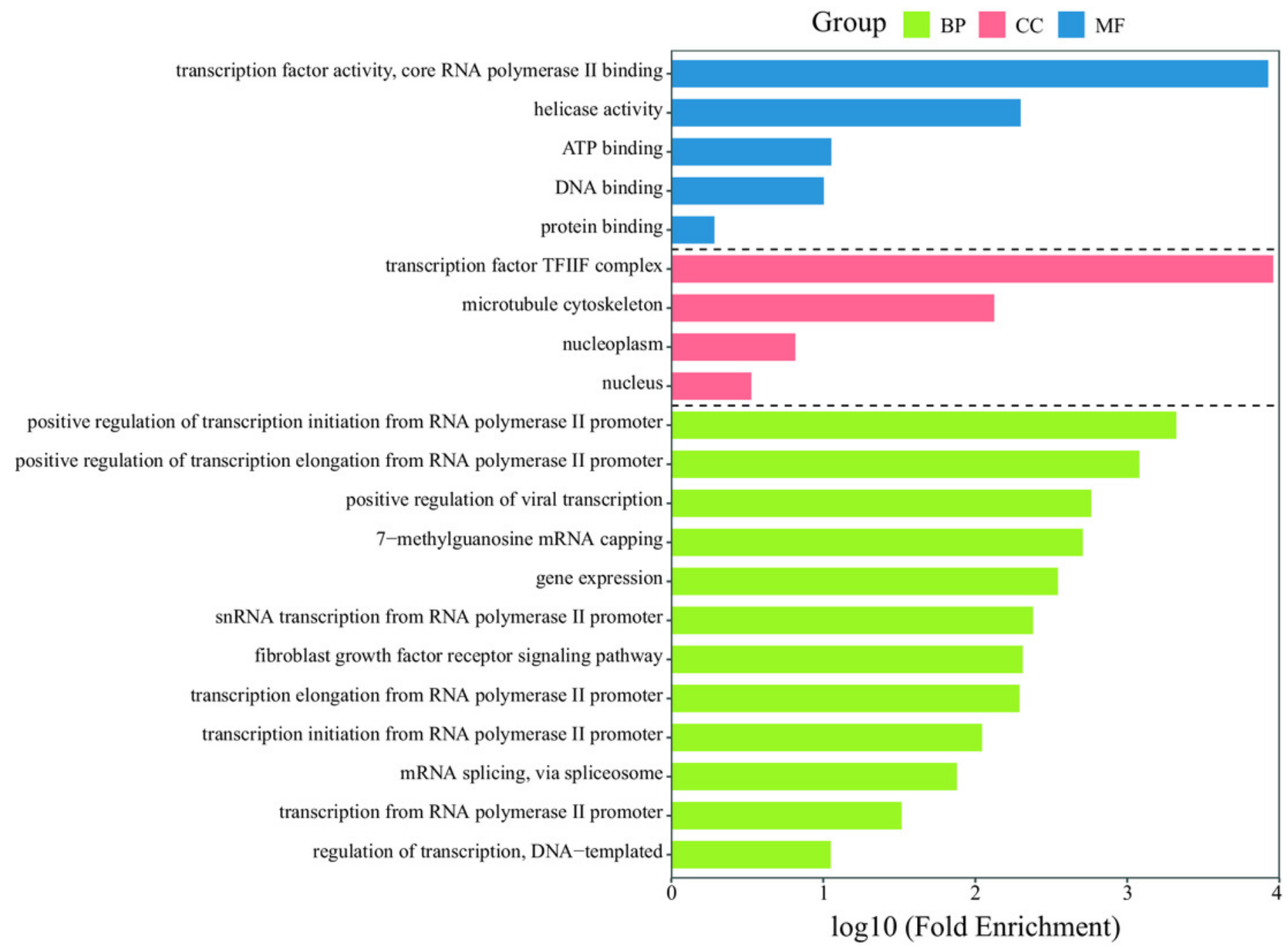


Figure 7

The expression levels of miR-708-5p and GTF2F2.

(A) The expression of miR-708-5p in paired lung tumor tissue samples and paraneoplastic tissue samples from the TCGA. (B) The expression of GTF2F2 in paired lung tumor tissue samples and paraneoplastic tissue samples from the TCGA. (C) The correlation between miR-708-5p and GTF2F2 mRNA expression in the TCGA dataset. 

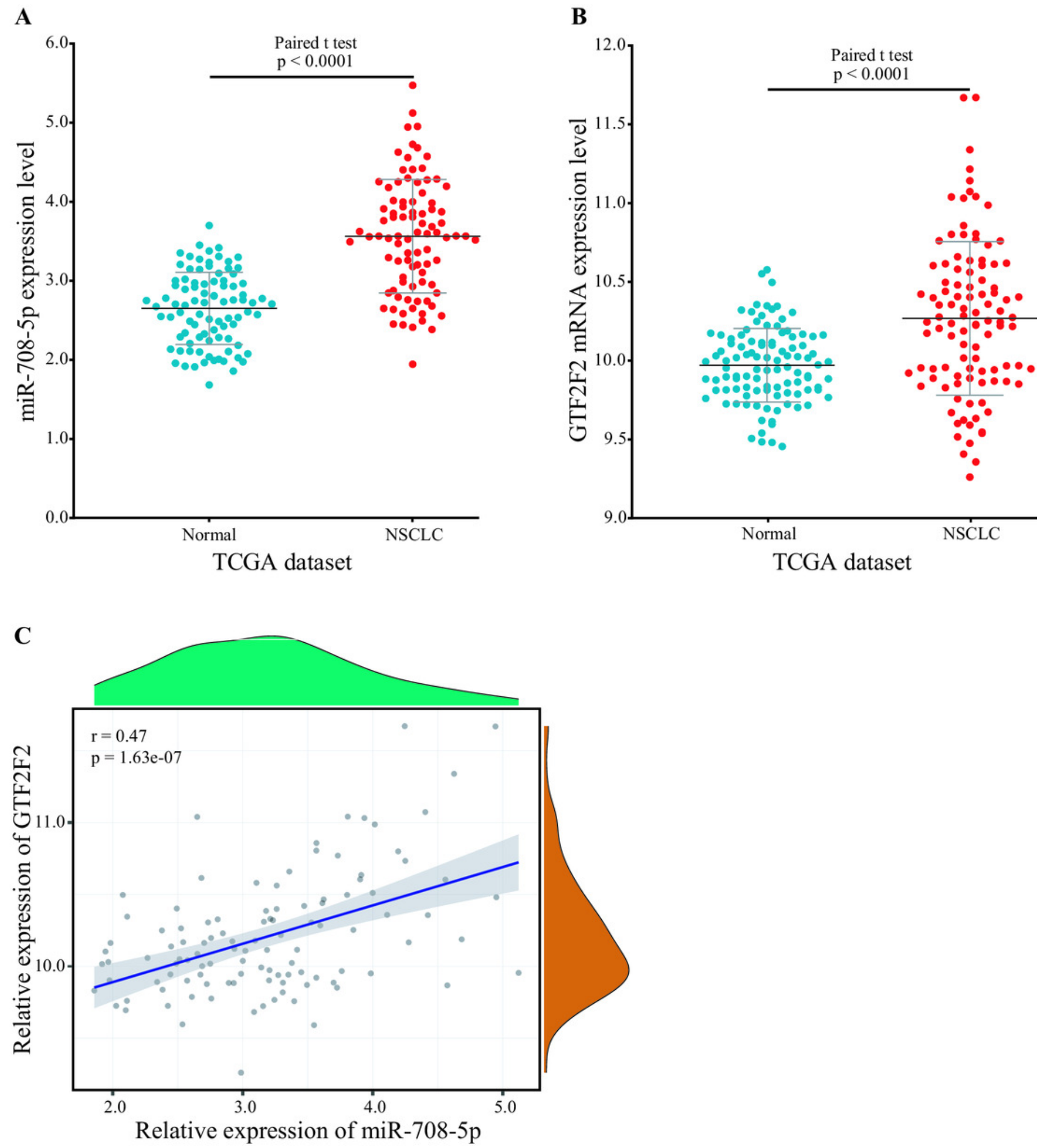
Figure 8

miR-708-5p expression levels and ROC curves in two external GEO datasets.

(A) The expression of miR-708-5p in the GSE56036 dataset. (B) The expression of miR-708-5p

in the GSE36681 dataset. (C) ROC curve of miR-708-5p in the GSE56036 dataset. (D) ROC curve of miR-708-5p in the GSE36681 dataset. 
A

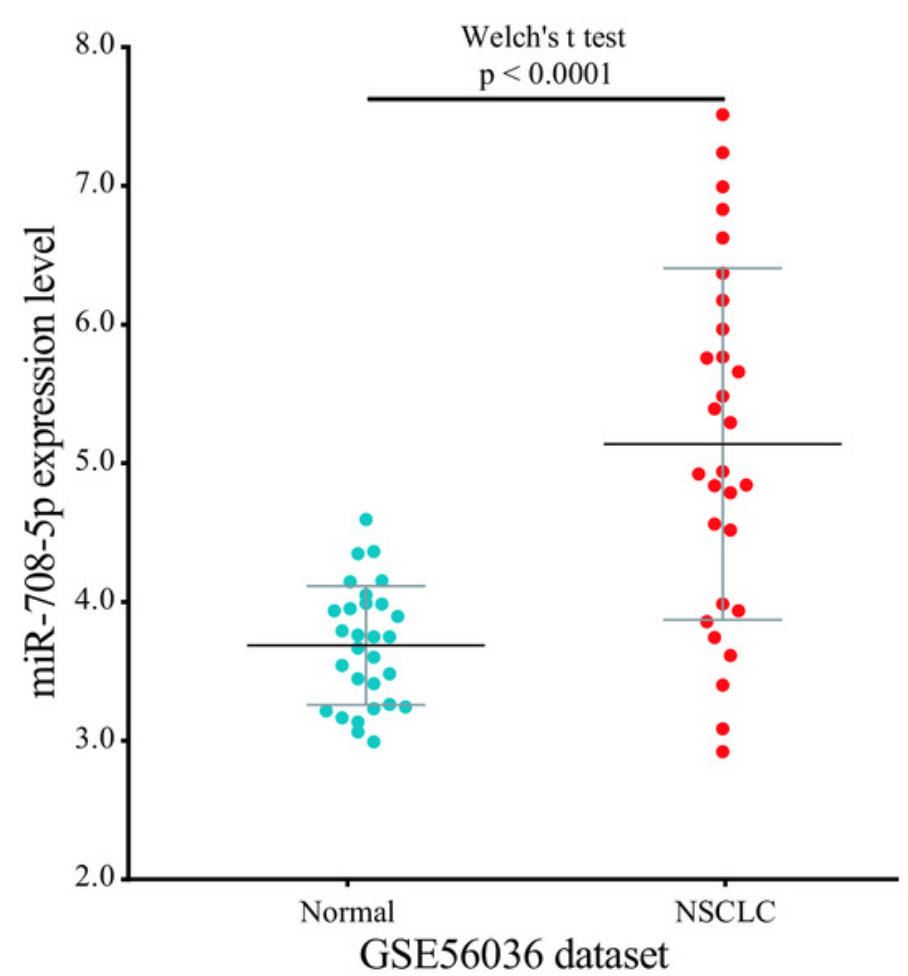

C

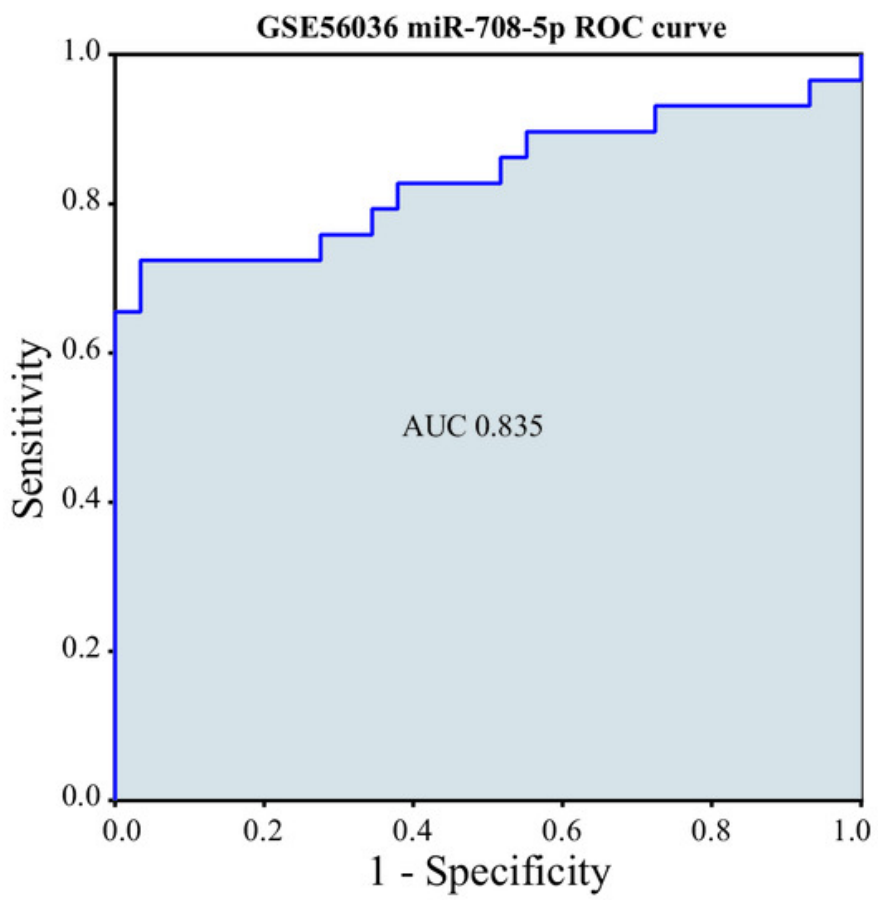

\section{B}

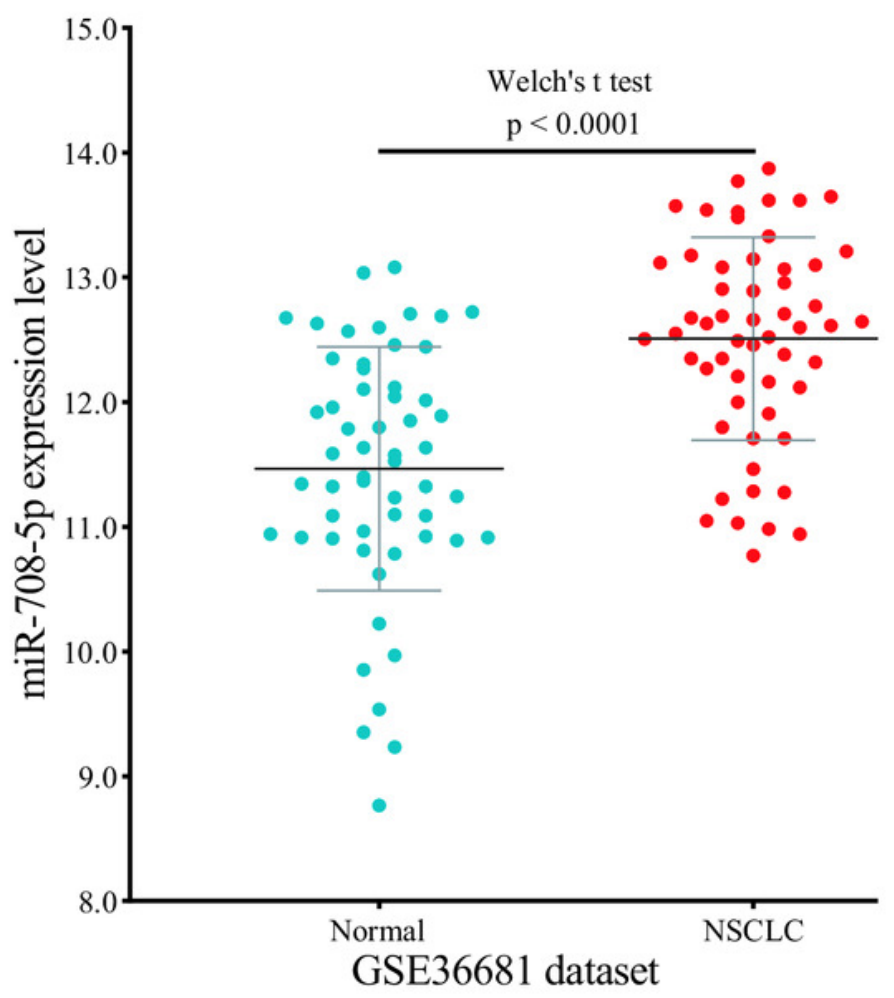

D

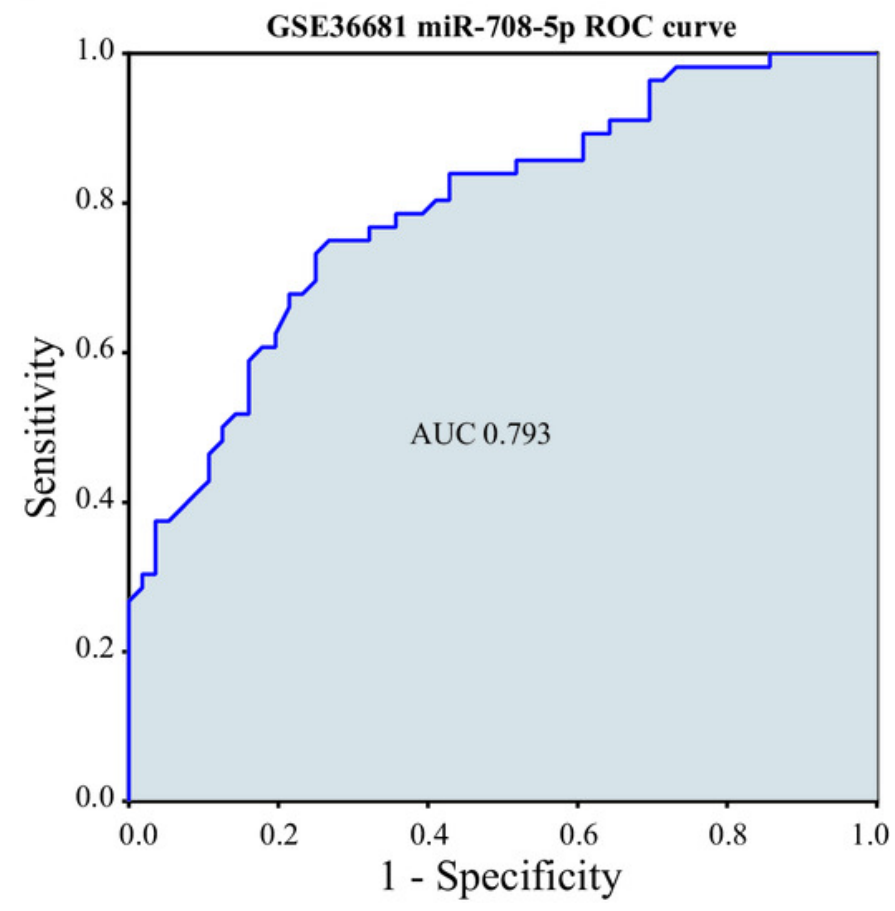




\section{Figure 9}

The schematic diagram of GTF2F2 related functions.

MB0 interacts directly with TFIIF, a general transcription factor that tightly associates with Pol II, enabling transcriptional elongation and accelerated tumor growth. TF2F2 binds to RMP to further inhibit the expression of pro-apoptotic gene $(B A X)$ and promote the expression of cell cycle-related genes (Cdk1 and Cyclin B1) to promote malignant tumorigenesis. Arrows indicate promotion and vertical bars indicate inhibition.

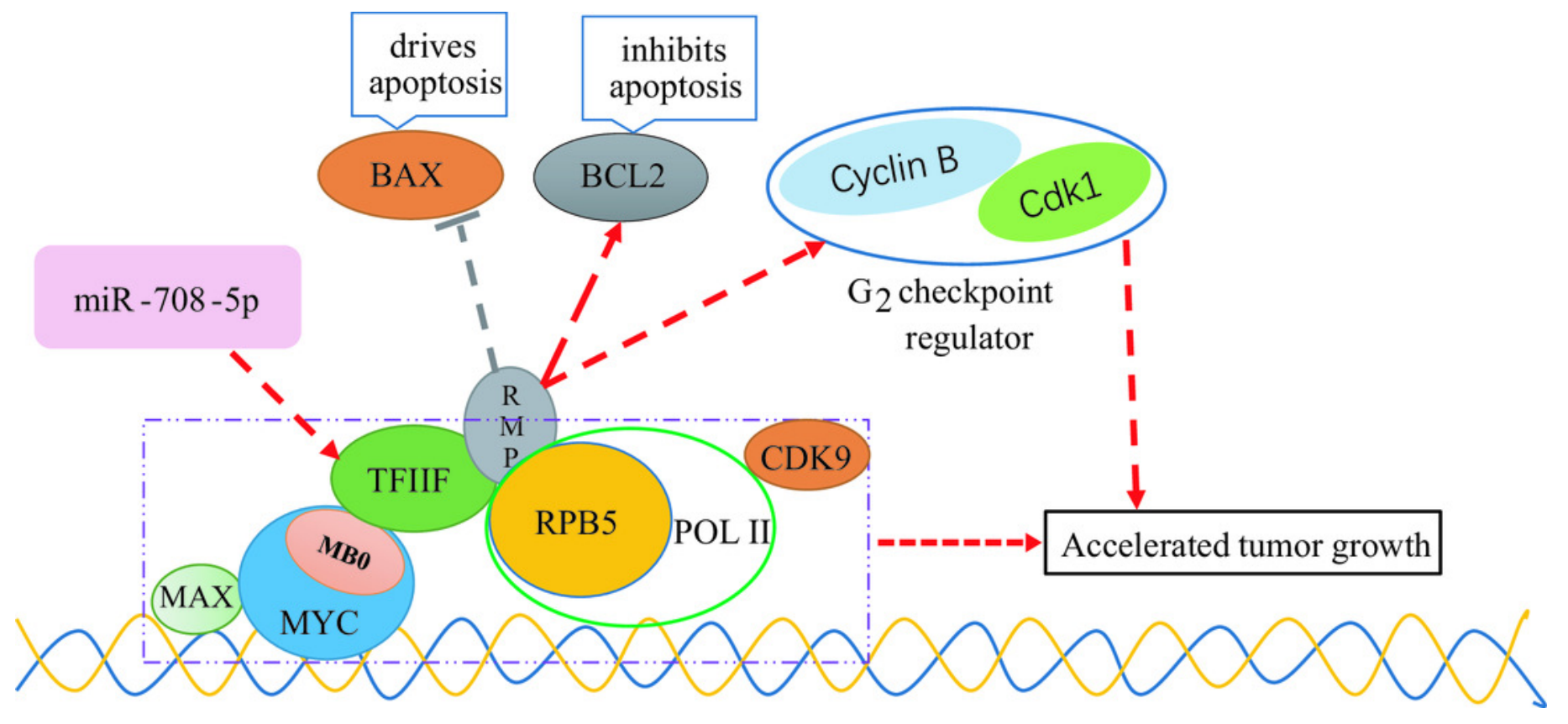


Table $\mathbf{1}$ (on next page)

Computational identification of 15 SDE miRNAs.

FC, Fold change; SNR, signal-to-noise. 
1 Table 1. Computational identification of 15 SDE miRNAs

\begin{tabular}{lllll}
\hline miRNA & P-value & log2 (FC) & SNR & Regulation \\
\hline hsa-miR-21-5p & $3.62 \mathrm{E}-87$ & 1.019878 & 1.991775 & Up \\
hsa-miR-30a-5p & $2.91 \mathrm{E}-202$ & -2.95875 & 1.503804 & Down \\
hsa-miR-96-5p & $1.13 \mathrm{E}-152$ & 2.589762 & 1.151469 & Up \\
hsa-miR-30d-5p & $2.28 \mathrm{E}-174$ & -1.8064 & 2.275344 & Down \\
hsa-miR-183-5p & $1.42 \mathrm{E}-144$ & 2.43937 & 1.126407 & Up \\
hsa-miR-210-3p & $4.49 \mathrm{E}-175$ & 4.419388 & 1.188131 & Up \\
hsa-miR-218-5p & $5.53 \mathrm{E}-217$ & -2.25307 & 1.9371 & Down \\
hsa-miR-1-3p & $1.94 \mathrm{E}-254$ & -2.53346 & 3.092538 & Down \\
hsa-miR-30b-5p & $6.69 \mathrm{E}-103$ & -1.35847 & 1.673761 & Down \\
hsa-miR-141-3p & $5.45 \mathrm{E}-125$ & 1.475862 & 1.273509 & Up \\
hsa-miR-144-3p & $3.63 \mathrm{E}-161$ & -3.18641 & 1.282499 & Down \\
hsa-miR-145-5p & $2.16 \mathrm{E}-111$ & -1.34576 & 1.850366 & Down \\
hsa-miR-126-3p & $9.74 \mathrm{E}-87$ & -1.43099 & 1.124842 & Down \\
hsa-miR-451a & $4.11 \mathrm{E}-149$ & -3.34021 & 1.172588 & Down \\
hsa-miR-708-5p & $2.22 \mathrm{E}-141$ & 3.087151 & 1.051665 & Up \\
\hline
\end{tabular}

2 FC, Fold change; SNR, signal-to-noise. 
Table 2 (on next page)

Results of the literature search of miR-708 in PubMed. 
1 Table 2. Results of the literature search of miR-708 in PubMed.

\begin{tabular}{llll}
\hline miRNA & Relevant literature & Cancer-related & Related to lung cancer \\
\hline miR-708-5p & 108 & 73 & 10 \\
\hline
\end{tabular}

2 


\section{Table 3 (on next page)}

Literatures about miR-708-5p associated with lung cancer in PubMed. 
1 Table 3. Literatures about miR-708-5p associated with lung cancer in PubMed

\begin{tabular}{llll}
\hline Year & Author & Journal & PMID \\
\hline 2019 & Yang, Xia et al. & Int J Biol Sci & 31360113 \\
2017 & Liu, Tianchi et al. & Clin Cancer Res & 28972040 \\
2016 & Hu, Ling et al. & Oncotarget & 26870998 \\
2015 & Wu, Xiaoping et al. & Oncotarget & 26678031 \\
2014 & Molina-Pinelo, Sonia et al. & PLoS One & 24625834 \\
2013 & Ryu, Seongho et al. & Cancer Cell & 23328481 \\
2012 & Jang, Jin Sung et al. & Clin Cancer Res & 22573352 \\
2012 & Pentheroudakis, George et al. & Clinical\&Experimental & 23124598 \\
2010 & Xing, Lingxiao et al. & Metastasis & 20526284 \\
2010 & Patnaik, Santosh K et al. & Mod Pathol & 20028859 \\
\hline
\end{tabular}

2 